\title{
REFLECTIONS
}

\section{Michelle's Story: The Complexity of Patient Care in a Family Medicine Residency Clinic}

\author{
Katbryn Justesen, MD \\ James D. Smith, MD, MPH \\ Katherine Swanson, DO, RN \\ Mark Yeazel, MD, MPH \\ James T. Pacala, MD, MS \\ Department of Family Medicine \\ and Community Health, University \\ of Minnesota Medical School, Minneapolis, \\ Minnesota
}

\begin{abstract}
Family medicine covers all ages and specializes in chronic disease management as well as acute care medicine. As the health of the population continues to grow in complexity, treating patients appropriately and efficiently is imperative to improving health outcomes and managing health care costs. Family medicine physicians are uniquely poised to provide this type of care. A patient story plus a look at the patients seen over the course of a day within a family medicine residency clinic explores the complexity of care and the ability of family medicine physicians to provide the necessary care. Taking a close look at who comes through our door on a particular day highlights 3 points: primary care physicians are seeing patients with an increasing complexity of needs, our society is witnessing an extreme increase in patients suffering with mental health problems and substance use disorders, and addressing social determinants of health must be part of the solution.
\end{abstract}

Ann Fam Med 2021;19:362-364. https://doi.org/10.1370/afm.2652.

\section{MICHELLE'S STORY}

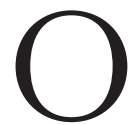
n August 5, 2019, Michelle, a long time patient of the University of Minnesota (UMN) Physicians Broadway Family Medicine (BFM) clinic, came in for an appointment soon after hospitalization for diabetic ketoacidosis (DKA). At age 42, she has struggled with managing her diabetes for over 10 years. Suffering a flare of chronic abdominal pain, she forgot what to do when she was ill and did not take any insulin. She developed DKA and was hospitalized in the intensive care unit (ICU). Throughout her hospital stay, including while in the ICU, her care was directed by a team of physicians from our clinic, including several who had met her before. Although knowledgeable about Michelle and her struggles thanks to prior multidisciplinary team continuity discussions, the resident physician that day for her clinic visit was meeting Michelle for the first time. The physician took a deep breath at the door prior to entering the exam room, resolving to show empathy and try to connect, and to take it one step at a time.

During her follow-up clinic visit, after she had spent some time thinking about her hospitalization, Michelle revealed that her life was spinning out of control. She shared the recent loss of a 17-year relationship that had been her most significant source of support. She confided that her depression, anxiety, chemical dependency, and post-traumatic stress disorder (PTSD) from a childhood of abuse were poorly controlled, and she was using cannabis almost daily to help cope. She struggles with the daily memories of her past trauma, which seep into every aspect of her life. She admittedly finds it difficult to prioritize her own health and medical needs, finding herself caregiving for everyone around her instead. Tearfully, she told her physician, "I want to be better, I just don't know how." 


\section{A TYPICAL DAY}

Michelle was just one of the patients seen on August 5, 2019, a routine day at the BFM clinic, a residency site of the UMN Department of Family Medicine and Community Health (DFMCH). The clinic is in an economically challenged neighborhood of Minneapolis where $60 \%$ of the households have a total income of less than $\$ 35,000$ and $68 \%$ of the clinic population is Black, a population that, due to centuries of racist policies and deeply pervasive systemic anti-Black racism today, suffers more than most. A day's work at BFM provides a snapshot of the current landscape of primary care.

On August 5, 89 patients were seen by 6 residents and 2 faculty clinicians. Appointments were generally scheduled every 20 minutes, although new patient visits, certain procedures, and hospital follow-up visits were 40 minutes. With Institutional Board Review approval at the University of Minnesota, we abstracted the electronic medical record to generate a profile of the patients served on a typical day at this primary care site. The patients ranged in age from 2 months to 92 years; 15 were aged $<10$ years. In the group of 74 patients aged $>10$ years, the mean number of chronic conditions was 3.8 with a range of $0-16 ; 72$ of these patients had at least 1 chronic condition and 69 were on at least 1 prescription medication (Table 1).

\section{THE COMPLEXITY OF CARE IN FAMILY MEDICINE}

What can we learn from the case of Michelle and this analysis of the day? Three themes emerge which emphasize the complexity of care provided in family medicine clinics. First, our insights reflect the increasing complexity of patients. The days of single problems such as sore throats or urinary tract infections are long gone. At BFM on August 5, there were only 9 adult visits in which a single condition was addressed, and 2 of those visits were for opioid use disorder, not

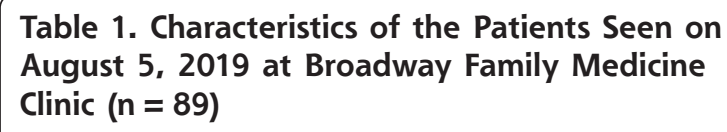

\section{Characteristic}

Frequency (\%)

$3+$ problems addressed at visit 49

$3+$ chronic conditions on problem list

At least 1 psychiatric diagnosis on problem list

On at least 1 psychiatric medication

Chronic or previous substance use disorder

Both psychiatric diagnosis and substance use disorder on problem list

At least 1 medical diagnosis plus 1 psychiatric or substance use disorder diagnosis exactly an uncomplicated problem. Some have suggested that primary care can be relegated to nonphysician clinicians, often providing care at pharmacy or retail clinics. ${ }^{1}$ Although these clinics have been shown to be effective at addressing single-problem, uncomplicated visits, we submit that the complex patients seen in modern primary care clinics are more appropriately served by physicians who are able to approach patient care with a whole person orientation and are trained to handle complex, ambiguous situations and have an interdisciplinary team available to assist with the care.

Medical complexity is notoriously hard to define or quantify. The overall agreement is only modest between models attempting to define complexity such as the Charlson Comorbidity Index or the Higashi score and what primary care physicians consider complex. $^{2}$ Primary care physicians identify complexity as multidimensional, with contributing factors such as medical illness, mental illness, socioeconomic challenges, and particular behavior traits. ${ }^{3}$ Additionally, history of trauma and adverse childhood experiences add to complexity. Regardless of the definition, the high rate of patients with comorbid medical and mental health diagnoses, often combined with substance use disorder and low socioeconomic status, typify this high degree of medical complexity in primary care.

The DFMCH sponsors 3 other residency training programs in Minneapolis/St Paul, serving medically complex patients from different demographic and cultural populations. East African immigrants, many with PTSD from a war-torn personal history, comprise over $25 \%$ of the patients at 1 clinic, and $42 \%$ of the patients at another clinic are Southeast Asian immigrants or firstgeneration Americans. Another clinic has added a lawyer to assist patients with legal matters, the vast majority of which relate to securing housing. Interpreters are required for $29 \%$ of the visits at these clinics. Although the challenges may differ across these sites, the 2 constants are the degree of patient complexity and the burden of disadvantaged social factors related to health.

Second, this analysis reflects the rise of mental health and chemical dependency problems in our society. According to the 2018 National Survey on Drug Use and Health, the prevalence of any mental illness and substance abuse disorder in adults were $19.1 \%$ and $8.5 \%$, respectively, in stark contrast to the $62 \%$ and $39 \%$ among the BFM patients seen on August 5. While part of this difference can be explained by the social and medical comorbidities experienced by the BFM patients, we believe they also depict a swiftly alarming increase in these disorders. National data from the Substance Abuse and Mental Health Services Administration notes a steady rise in mental illness diagnoses between 2008 and 2018 with the greatest increase in 
people aged 18 to 25 years, with a rise from $18.5 \%$ in 2008 to $26.3 \%$ in $2018 .{ }^{4}$ Primary care clinicians validate that mental health and substance abuse disorders have increased dramatically in the past 3 years. In addition, the COVID-19 pandemic will likely amplify preexisting mental health disorders and perhaps lead to more diagnoses. We believe updated population-based data will confirm this burgeoning problem, and that policy makers and health care systems should plan accordingly.

Third, our experience highlights the importance of addressing social determinants of health, which permeate the context of health and health care. Factors such as financial challenges, limited transportation, food insecurity, and low health literacy have a profound influence on health. Social determinants influence the health of a person with diabetes who is limited in food and medication options due to cost, or a person on warfarin who relies on public transportation to receive frequent lab monitoring. Psychosocial factors, family stressors, and lack of social supports can make coping and managing chronic disease or sobriety challenging. The recent COVID-19 pandemic and events surrounding the killing of George Floyd have only further exacerbated the adverse effects of such factors on our patients.

Although family medicine physicians may not be able to primarily address all of these issues, employing team care models featuring health professionals such as social workers and care coordinators can fulfill this critical need.

\section{PROVIDING CARE FOR PATIENTS WITH COMPLEX NEEDS}

Providing care in the context of the complexities outlined above can be daunting — the resident seeing Michelle was understandably overwhelmed-a common experience in family medicine. There was so much to cover-where to start? Michelle's diabetes needed to be addressed, but it had been out of control for 10 years. How might this time be different? And what was the root cause? Michelle forgot to take her insulin because of her abdominal pain, which had been worked up extensively a number of times, without evidence of an organic cause. What about her mental health and substance use? Her traumas that bubble to the surface whenever her life gets complicated? How to sort out these problems, the stuff of incrementalism, when such instabilities also lead to infrequent follow-up?

Michelle's story is not unusual at BFM, whose patients present with complex medical issues compounded by mental health challenges that are chronically worsened by a myriad of social factors and systemic racism. Meting out the care of these complex patients to a multitude of specialists, each focusing on a single problem, can contribute to problems such as polypharmacy, iatrogenic illness, lack of care coordination, and overall inefficiency. Such an approach works against the goal of patient-centered care. The increasing complexity of these patients warrants further investment in the type of care offered at BFM: full-spectrum family medicine offering a wide range of integrated services, including behavioral health services, substance use disorder treatment, pharmacy expertise, care coordination, and continuity of care with a single clinician. Further validation is found in multiple studies demonstrating improved outcomes for patients with a primary care physician, providing evidence of the value and effectiveness of primary care. ${ }^{5,6}$

As family physicians, we, like other primary care clinicians, embrace our patients' complexities. We strive to provide a safe environment where patients like Michelle feel comfortable sharing their stories. Only by digging into these stories are we able to create and nurture relationships of mutual trust, understanding, and respect. These relationships take time and patience to build and foster what Atul Gawande has termed the "heroism of the incremental" — primary care that improves health, lowers costs, and is ultimately fulfilling for patients and for doctors. As family physicians, although we are uncomfortable with Gawande's hero label, we endorse his call to shift the focus of health policy to promoting effective primary care approaches for patients like Michelle who present as a "medical emergency."

To read or post commentaries in response to this article, go to https://www. AnnFamMed.org/content/19/4/362/tab-e-letters.

Key words: delivery of health care; patient-centered care.

\section{References}

1. Dalen JE, Ryan KJ, Alpert JS. More sub-subs are coming! Am J Med. 2019;132(2):132-133. 10.1016/j.amjmed.2018.07.031.

2. Grant RW, Ashburner JM, Hong CS, Chang Y, Barry MJ, Atlas SJ. Defining patient complexity from the primary care physician's perspective: a cohort study. Ann Intern Med. 2011;155(12):797-804. Erratum: Ann Intern Med. 2012;157(2):152.

3. Loeb DF, Binswanger IA, Candrian C, Bayliss EA. Primary care physician insights into a typology of the complex patient in primary care. Ann Fam Med. 2015;13(5):451-455.

4. Substance Abuse and Mental Health Services Administration. Accessed Aug 5, 2020. https://www.samhsa.gov/data/report/20162018-nsduhnational-maps-prevalence-estimates-substate-region

5. Basu S, Berkowitz SA, Phillips RL, Bitton A, Landon BE, Phillips RS. Association of primary care physician supply with population mortality in the United States, 2005-2015. JAMA Intern Med. 2019; 179(4):506-514. 10.1001/jamainternmed.2018.7624

6. Chang $\mathrm{CH}$, Stukel TA, Flood AB, Goodman DC. Primary care physician workforce and Medicare beneficiaries' health outcomes. JAMA. 2011;305(20):2096-2104. 10.1001/jama.2011.665.

7. Gawande A. The heroism of incremental care. The New Yorker. Published Jan 15, 2017. https://www.newyorker.com/magazine/2017/01/ 23/the-heroism-of-incremental-care 